\title{
Virtual User Interface
}

Shreyas S.

Student/DAEI/Rajagiri School of Engineering \& Technology Kerala, India shreyasshankar@yahoo.com

\section{Sukanya R. Warier}

Faculty /DAEI/Rajagiri School of Engineering \& Technology Kerala, India sukanyarw@rajagiritech.ac.in

\section{Veena S.}

Student/DAEI/Rajagiri School of Engineering \& Technology Kerala, India veena2961@gmail.com

\section{Vineeth Domini K.}

Student/DAEI/Rajagiri School of Engineering \& Technology Kerala, India vineethdomini06@gmail.com

\section{Stephy Caroline}

Student/DAEI/Rajagiri School of Engineering \& Technology Kerala, India stephycaroline94@gmail.com

\section{Shanmuga Priya}

Faculty /DAEI/Rajagiri School of Engineering \& Technology Kerala, India priyams@rajagiritech.ac.in

\begin{abstract}
In this world of technology, the interface between man and machine is very useful in making things easier and is on ascend. This can be of great use in almost all sectors, more specifically the education field where we can make use of this for teaching and other applications in a way to improve and make class sessions more interesting for students. This is a world of technology, paved way for smart boards, which made these work easier. Nevertheless, these solutions to problems are of high cost, thus making their movement slow, in most of the countries. In these circumstances, we present our paper of Virtual User Interface, which consists of minimum hardware requirements of low cost with help of free open software available. The hardware consists of an IR-cam and a pointing device to detect the user interactions on a surface and the system is compatible with any video projectors. We truly believe that the proposed system will represent an important contribution which enables users to interact with machines quiet easily on a widespread basis with all the benefits.
\end{abstract}

Keywords: IR Pen, Raspberry Pi, IR Camera, Virtual user interface.

\section{INTRODUCTION}

The rapid changes occurring in information and communication technologies have changed the traditional classroom environment and teaching methods. Projectors, Internet linked computers in classrooms, mobile phones, digital cameras and video recorders affect many aspects of education ranging from student projects to lesson presentations. Another aspect of the last 20 years has been the interactive whiteboard which consists of a connection between a computer, a projector and a touch screen electronic whiteboard. These solutions are usually expensive, making their acceptance slow. In this context, we present an open-source interactive whiteboard along with low-cost hardware requirements. Here, we developed a low cost virtual interface using easily available hardware devices namely, a Raspberry-Pi with an infra red camera, a video projector and an IR pointing device such as LED pen.

In the current scenario, the existing system for presentation and teaching aids is the chalk and board system. The problems faced in this system are that it may cause dust allergies and it may also make the system less interactive and the class sessions boring. Also while using markers to write we need to replace it each time it gets over. In short, it's an ineffective and inefficient way of approach. To replace this and make the system more advanced and the classes more interactive, we implemented the VUI.

\section{REQUIREMENT ANALYSIS}

Requirements analysis and validation is a process of refinement, modelling and specification of the already discovered user requirements. 


\subsection{User Requirements}

On the basis of requirement survey conducted among the users, we have reached to a conclusion that more than $90 \%$ of the students are interested to attend the classes in a smart classroom. $95 \%$ of teachers stated that projector and interactive board made their teaching more efficient and effective. $80 \%$ people believe that interactive boards will enhance the student abilities. Another feature people wanted was to make the interactive board touch sensitive.

\subsection{Project Requirements}

On the basis of the requirements demanded by the user the following project requirements were found out:

- Reduce the cost of interactive tool.

- Compatible with traditional projectors.

- High clarity and visibility of the board.

- Additional facilities like drawing tool, internet connectivity etc.

\subsection{Specific Requirements}

While working as teaching aid, only a projector and the standalone embedded computer unit is required. While used as presentation aid, it requires the embedded computer unit along with a computer as the video source. Software interfaces for communication between the IR sensor and the raspberry pi is required and also a software interface should be developed for the communicating RF signals from the pointing device.TCP/IP, UDP protocols are used for communication.

\section{DESIGN}

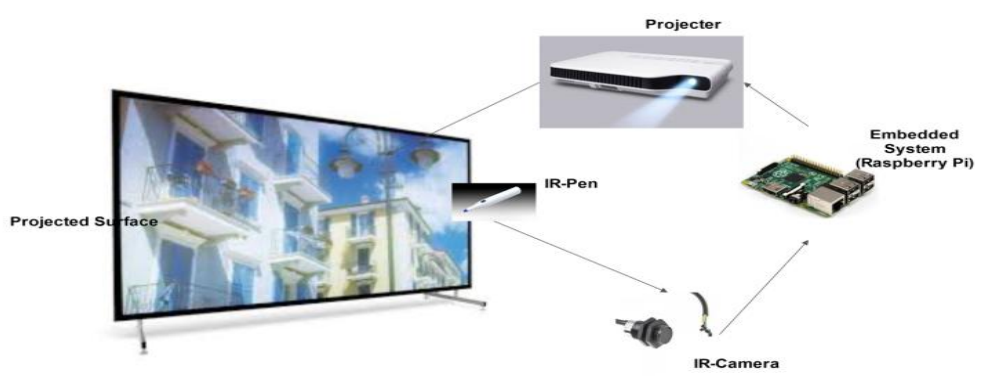

Fig1. System Overview

The system has been designed to serve both as an electronic whiteboard and wireless desktop mirroring device. For implementing the touch interface, pointing device along with IR cam is used. The pointing device is enabled with a left click and right click button. With each button press a unique digital code is transmitted, this code is mapped to corresponding left click and right clicks in mouse driver. Also the coordinates of clicked position is identified by the IR cam and the suitable transformations are applied to obtain the relative coordinates with the edges of the projected board.

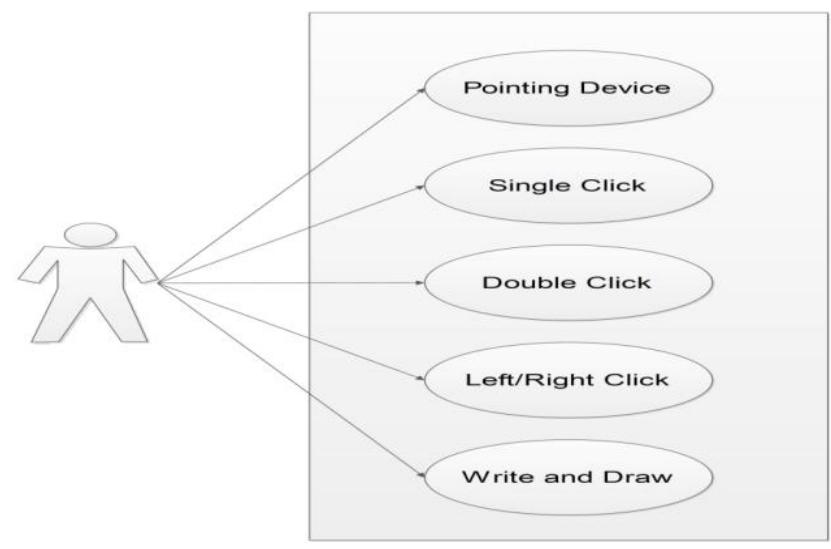

Fig2. IR Pen 


\subsection{User Perspective}

Numerous studies have shown that use of VUI improves learning processes, specifically where the integration between the teacher's instruction style and the VUI's potential enables meaningful instruction. Students reported that the use of the VUI enhances motivation to learn, raises the level of concentration, improves behavior, and enhances learning because it is fun and innovative. Students who learned with VUI were more attentive and engaged in learning, participated more actively in the classroom and interacted much more with their teachers. Additional studies provided the evidence that the VUIs serve a significant motivational tool for students. Criticisms regarding the use of VUIs were the technical problems that it is difficult to see the boards from a distance, and the teachers are not skilled enough in their use of the VUI.

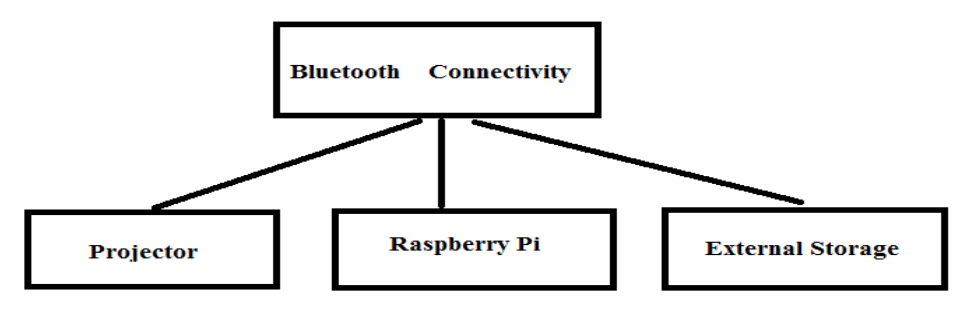

Fig3. Embedded System

\subsection{System Architecture}

The hardware components of the system include embedded computer, IR cam, pointing device and the projector. We are going to work in Linux platform. The library NumPy is referred for matrix transformations. The overall system can be used as interactive white board as well as a wireless mirror server.

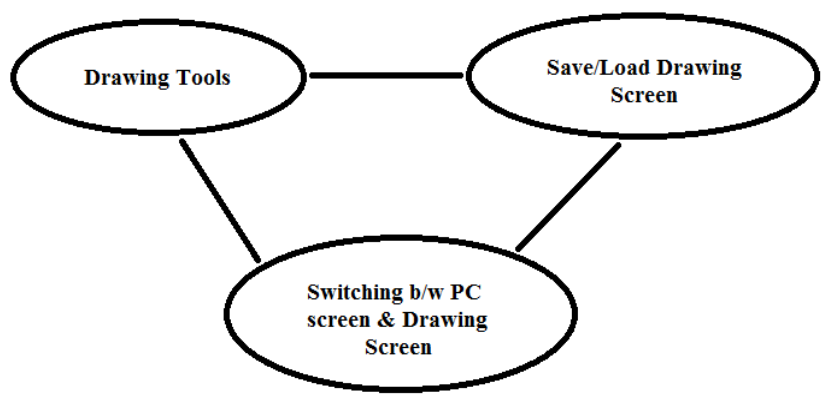

Fig4. System Interaction

\subsection{Sequence Diagram}

A Sequence diagram is an interaction diagram that shows how processes operate with one another and in what order. It is a construct of a Message Sequence Chart. A sequence diagram shows object interactions arranged in time sequence. It depicts the objects and classes involved in the scenario and the sequence of messages exchanged between the objects needed to carry out the functionality of the scenario. Sequence diagrams are typically associated with use case realizations in the Logical View of the system under development. Sequence diagrams are sometimes called event diagrams or event scenarios.

\subsection{Graphical User Interface}

In the user interface there are four options for the user:-

1. Connect: - this is for connecting with the camera.

2. Disconnect: - this is for disconnecting with the camera.

3. Calibrate: - this is for starting the calibration process.

4. Paint window: - this option will open a new dialogue box i.e., a drawing interface 


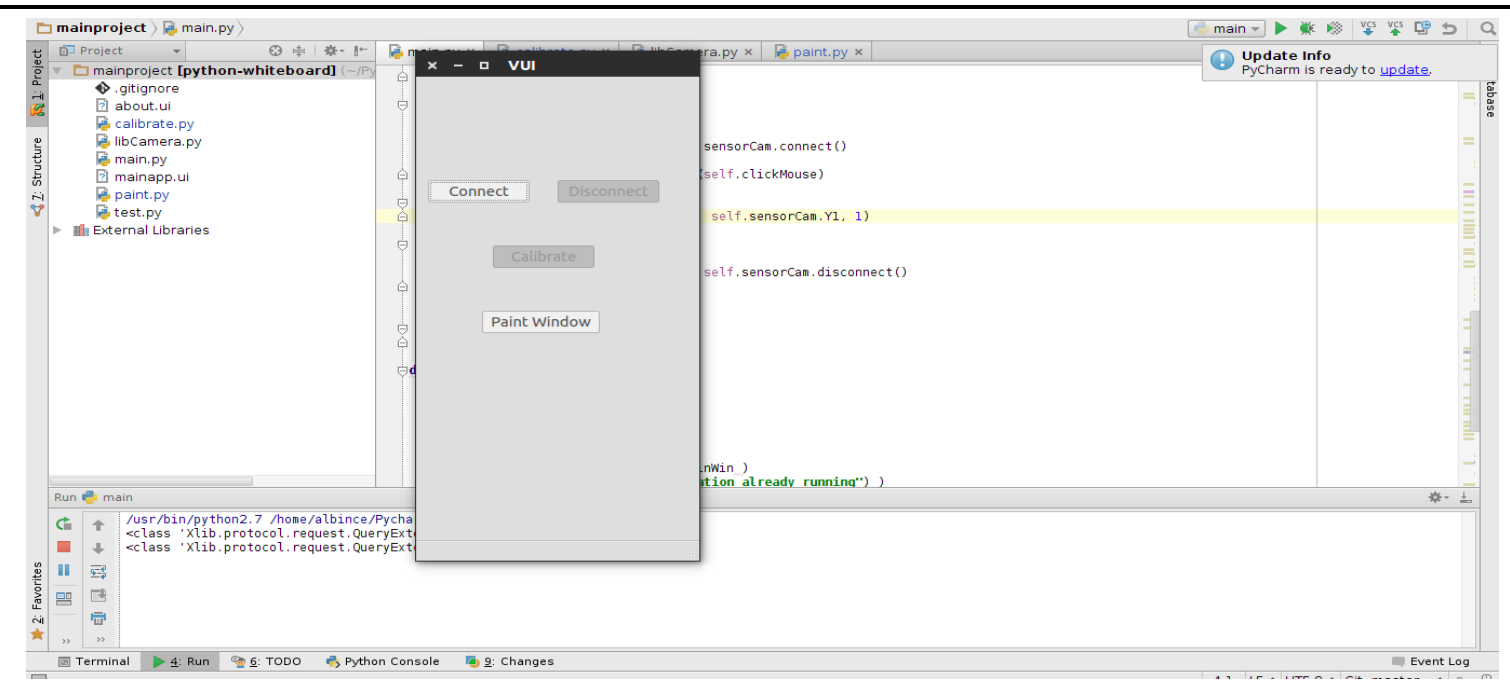

Fig5. User Interface

\subsection{Calibration}

When the user select the calibrate option from the graphical user interface he has to fix the end points of the projected screen. This is done by touching on the four corner points using the IR pen. Initially user must select the left upper point $(40,40)$ and proceed in the clock wise direction. The top most right is considered as (40, width-40) and bottom left is (height-40, 40). These 4 points are arbitrary. This phase can easily completed by simply touching on the blinking points. A linear equation is formed from this and the coefficients are taken and made as a matrix.

Now onwards, the user must be take care to touch inside this window, otherwise his touch will be invalid.

(a)

Fig6. Calibration Screen

\subsection{Virtual Touch Interface}

The user can project any contents in their device and can perform the operations like left click, right click using the IR pen, on the projected surface. This will enhance the teaching and presentation practices.

By using the drawing tool, user can highlight and mark the important ideas on the screen, later they can be saved and retrieved as notes to the students.

\subsection{Drawing Interface}

When user selects the option paint window from the graphical user interface he will get into the drawing interface. This is similar to any other drawing software like MS paint, Corel painter etc.

Here we have a drawing area, menu bar and tool bar.

1. Menu bar - containing options for saving and loading image

2. Tool bar - various colours can be selected, brush size can be varied. 


\section{Testing}

Testing is an essential step in the development of Virtual User Interface where errors are detected and corrected. Here we have included all the possible test cases to ensure that the system is implemented correctly. There are two main approaches for testing.

- Black box testing - White box testing

\subsection{Black Box Texting}

Black-box testing is a method of software testing that tests the functionality of an application as opposed to its internal structures or workings (see white box testing). Specific knowledge of the application's code or internal structure and programming knowledge in general is not required. The tester is only aware of what the software is supposed to do, but how i.e. when he enters a certain input, he gets a certain output; without being aware of how the output was produced in the first place. Test cases are built around specifications and requirements, i.e., what the application is supposed to do. It uses external descriptions of the software, including specifications, requirements, and designs to derive test cases. These tests can be functional or non- functional, though usually functional. The test designer selects valid and invalid inputs and determines the correct output. There is no knowledge of the test object's internal structure. This method of test can be applied to all levels of software testing: unit, integration, system and acceptance.

\section{Advantages}

- As the tester has knowledge of the source code, it becomes very easy to find out which type of data can help in testing the application effectively.

- It helps in optimizing the code.

- Extra lines of code can be removed which can bring in hidden defects.

- Due to the tester's knowledge about the code, maximum coverage is attained during test scenario writing.

\section{Disadvantages}

- Due to the fact that a skilled tester is needed to perform white box testing, the costs are increased.

- Sometimes it is impossible to look into every nook and corner to find out hidden errors that may create problems as many paths will go untested.

- It is difficult to maintain white box testing as the use of specialized tools like code analysers and debugging tools are required.

\subsection{White Box Texting}

White-box testing is a method of testing software that tests internal structures or workings of an application, as opposed to its functionality (i.e. black- box testing). In white-box testing, internal perspectives of the system, as well as programming skills are used to design test cases. The tester chooses inputs to exercise paths through the code and determine the appropriate outputs. This is analogous to testing nodes in a circuit, e.g. in-circuit testing (ICT). While white-box testing can be applied at the unit, integration and system levels of the software testing process, it is usually done at the unit level. It can test paths within a unit, paths between units during integration, and between subsystems during a system level test. Though this method of test design can uncover many errors or problems, it might not detect unimplemented parts of the specification or missing requirements. As part of white box testing, assessment of code coverage is tested and verified. The test cases are executed to ensure maximum code coverage. Code coverage is a measure used in software testing. It describes the degree to which the source code of a program has been tested. It is a form of testing that inspects the code directly and is therefore a form of white box testing.

\section{Advantages}

- Well suited and efficient for large code segments.

- Code access not required.

- Clearly separates user's perspective from the developer's perspective through visibly defined roles. 
- Large numbers of moderately skilled testers can test the application with no knowledge of implementation, programming language or operating systems.

\section{Disadvantages}

- Limited coverage since only a selected number of test scenarios are actually performed.

- Inefficient testing, due to the fact that the tester only has limited knowledge about an application.

- Blind coverage, since the tester cannot target specific code segments or error prone areas.

- The test cases are difficult to design.

\section{Conclusion}

As specified by the requirements, the hardware has been completed successfully. We developed an embedded system with optimal use of hardware, which provides an interactive touch interface at a considerable cost. We believe that the proposed solution will represent a valuable contribution to ease the access in the user interface and increase widespread use of the solution with obvious benefits.

We placed the IR camera about $50 \mathrm{~cm}$ from the screen at an angle of 30 degree, which created an IR field. As the changes were made by the IR pen, it was detected by the IR camera and the corresponding code was run and the setup was implemented.

In the present context, there are several similar systems available, but they are costly and people with fragile economy cannot afford such systems. Our project provides more flexible and friendly interface with very low cost. The concept of Virtual User Interface is a major step towards the development of smart classrooms and interactive conference halls. The ease of presentation and user interaction will make a significant change in the field of education, finance and other day to day activities. The future enhancements of the proposed system that we have planned are to extend the proposed system to all platforms and operating systems.

\section{ACKNOWLEDGEMENT}

We have put all our effort and time in completing this project research work successfully. However, it would not have been possible without the support, encouragement, guidance and help of our kind hearted teachers. We would also like to extend our sincere gratitude to our Management, Rajagiri School of Engineering \& Technology for their co-operation and support which helped us a lot.

\section{REFERENCES}

[1] Shuai Zhang, Wenrui He, Qiao Yu and Xiaojuan Zheng, Low-cost interactive white- board using the Kinect, pages 1-5, Image Analysis and Signal Processing (IASP), International Conference on 9-11 Nov. 2012.

[2] www.raspbian.org, Raspbian, 2014.

[3] Mark Summerfield, Rapid GUI Programming with Python and QT.

[4] Chao-Hsuing Tseng, Yung-Chiang Wei, Design and implementation of the Multi- Touch Tables.

[5] Gigabit Ethernet Based Image Acquisition System for IR Camera, Sixth International Conference on Computational Intelligence and Communication Networks, 2014.

[6] Internet of Things approach for motion detection with Raspberry Pi, International Conference on Intelligent Computing and Internet of Things (ICIT), 2015

[7] Think Python, O'Reilly, 2005

[8] Getting started with Raspberry Pi, O’Reilly, 2013.

[9] www.numpy.org/scipy.org 